\title{
Gesammelte Studien zum Alten Testament
}

\author{
Hans-Peter Mathys
}

Walter Dietrich, Von David zu den Deuteronomisten. Studien zu den Geschichtsüberlieferungen des Alten Testaments (Beiträge zur Wissenschaft vom Alten und Neuen Testament 156). Verlag Kohlhammer, Stuttgart/Berlin/Köln 2002, 280 S. James Alfred Loader, Begegnung mit Gott (Wiener Alttestamentliche Studien 3). Verlag Peter Lang, Frankfurt a.M. usw. 2001, 410 S. - Rudolf Mosis, Gesammelte Aufsätze zum Alten Testament (forschung zur bibel 93). Echter Verlag, Würzburg 1999, 396 S. - Ludwig Schmidt, Gesammelte Aufsätze zum Pentateuch (Beihefte zur Zeitschrift für die alttestamentliche Wissenschaft 263). Verlag Walter de Gruyter, Berlin/New York 1998, 286 S. - Rudolf Smend, Die Mitte des Alten Testaments. Exegetische Aufsätze. Mohr Siebeck, Tübingen 2002, 279 S.

Fünf Bände mit gesammelten Aufsätzen über das Alte Testament gilt es im folgenden vorzustellen. Sie könnten unterschiedlicher nicht sein; die Rezension trägt dem Rechnung.

1. Im Jahre 1986 publizierte Rudolf Smend unter dem Titel »Die Mitte des Alten Testaments« zwei kürzere Monographien und eine Reihe von Aufsätzen, welche die Forschung, weit über die Fachgrenzen hinaus, stark bestimmten und noch bestimmen. Im Jahre darauf erschien ein zweiter Band mit gesammelten Schriften »Zur ältesten Geschichte Israels«, und 1991 schließlich ein dritter Band mit wissenschaftsgeschichtlichen Arbeiten: »Epochen der Bibelkritik«. Alle drei Bücher erschienen in der Reihe »Beiträge zur evangelischen Theologie« (Bd. 99; 100; 109). Hier ist nun der erste dieser Bände, neu bei Mohr Siebeck herausgegeben, zu besprechen. Er enthält gegenüber der Erstauflage gewichtige Änderungen und trägt deshalb zu Recht einen neuen Untertitel: »Exegetische Aufsätze«. Der Beitrag über Karl Barth (»Nachkritische Schriftauslegung«) wurde gestrichen, dafür wurden zwei Aufsätze aus Bd. 2 übernommen: »Das uneroberte Land« sowie »Der biblische und der historische Elia«. Schließlich ist S.s Beitrag in dem zusammen mit U. Luz verfaßten Büchlein »Gesetz« (Biblische Konfrontationen, 1981) aufgenommen. So umfaßt der Band jetzt - neben den bereits genannten Titeln - folgende Arbeiten: »Die Bundesformel«; »Die Mitte des Alten Testaments«; »Theologie im Alten Testament«; »Elemente alttestamentlichen Geschichtsdenkens«; »Das Gesetz und die Völker. Ein Beitrag zur deuteronomistischen Redaktionsgeschichte«; »Der Ort des Staates im Alten 
Testament«; »Das Wort Jahwes an Elia. Erwägungen zur Komposition von 1Kön 17-19«; »Das Nein des Amos«; »Das Ende ist gekommen . Ein Amoswort in der Priesterschrift«; »Zur Geschichte von האמין/; »Essen und Trinken - ein Stück Weltlichkeit des Alten Testaments«. Abgeschlossen wird der Band (nach den bibliographischen Nachweisen) mit Registern: Bibelstellen, Autoren, Stichworte (neu). Über Qualität und Bedeutung dieser Beiträge brauchen keine Worte verloren zu werden; ein Sammelband, der eine Neuauflage erlebt, hat seine Berechtigung erwiesen, die bei diesem Genus nicht immer gegeben ist. Besonders wichtig ist der Beitrag "Das Gesetz im Alten Testament«, der eine der schönsten (Überblicks-)Darstellungen zu diesem Thema bildet und zu Recht in den Sammelband aufgenommen worden ist; dieser hat durch diese und die übrigen Änderungen gegenüber seinem Vorgänger an Geschlossenheit gewonnen. S., der ein blendendes Deutsch schreibt, ist wie sein Lehrer A. Alt ein Mann der kurzen Form; er versteht es, auf zwanzig oder dreißig Seiten lesbar und schön zu formulieren, was andere zu einer Monographie auswälzten. Alles wirkt leicht und selbstverständlich, so daß man gelegentlich in Gefahr steht, wichtige Aussagen zu überlesen. Der Band - unverzichtbar für jeden Alttestamentler - eignet sich aùsgezeichnet als Weihnachtsgeschenk für Studenten, denen man das Alte Testament schmackhaft machen will.

2. Walter Dietrich geht im Vorwort zu seinem Sammelband recht ausführlich und beinahe konfessorisch auf seine Arbeit ein: »In seltsamer Hartnäckigkeit hat mich das Leben, haben mich innere Neigung und von außen kommende Anforderungen immer wieder an den doppelten Themenkreis 〉David` und `Deuteronomismus` herangeführt.« (8) D. spricht an dieser Stelle von einem wissenschaftlichen Schicksal, und hinter dem Leben kann man Gott erkennen. Im einleitenden, hier erstmals publizierten Aufsatz »Das biblische Bild der Herrschaft Davids« versucht er den Nachweis zu erbringen, daß dieser König in allen Punkten dem Herrscherbild des Alten Orients entspricht, sich aber auch deutlich von diesem abhebt. »Gerade dieses >Mehr (und auch >Wenigers) ist es, was ihn ... zur einzigartigen Leitfigur der jüdisch-christlich geprägten Kultur- und Geistesgeschichte werden lassen konnte.« (23) Vor noch nicht allzu langer Zeit hätte man für dieses Mehr und Weniger Ausdrücke wie »Überlegenheit« oder »Einzig(artig)keit« verwendet; das ist theologisch heute nicht mehr opportun. D. geht in seinen Arbeiten von folgenden Sätzen aus: Gott hat mit seinem Volk in der Geschichte gehandelt. Dieses Handeln findet seinen Niederschlag in den Dokumenten, die von ihm berichten. Literarkritik bedeutet für $\mathrm{D}$. die Möglichkeit, Gottes Wirken nachzuzeichnen. Es erstaunt deshalb nicht, daß er Literarkritik sowie Redaktionsgeschichte mit viel Freude und Eifer be- 
treibt; sie ist ein Kerngeschäft der alttestamentlichen Wissenschaft und ein theologisches Unternehmen.

Nachdem R. Smend mit seinem Aufsatz »Das Gesetz und die Völker. Ein Beitrag zur deuteronomistischen Redaktionsgeschichte« den Startschuß zur Entwicklung des Göttinger Deuteronomismusmodells, wonach mit verschiedenen Deuteronomisten zu rechnen ist, gegeben hatte, erweiterte D. dieses mit DtrP. Mit T. Veijola zusammen gehört er zu den überzeugtesten Göttingern. Seine Aufsätze enthalten relativ wenig Auseinandersetzung mit dem Cross'schen Alternativmodell, das mit Fortschreibungen operiert, oder mit noch neueren Entwürfen und Ansätzen, etwa Westermanns oder Knaufs, die - gegen M. Noth - die Existenz eines vom Deuteronomium bis zum Zweiten Königebuch reichenden deuteronomistischen Geschichtswerks bestreiten. Diese Zurückhaltung ist begreiflich: Man kann sich nicht liebevoll und detailliert mit dem Innenausbau eines Hauses beschäftigen und gleichzeitig erwägen, welch anderen Grundriß man für es auch hätte wählen können.

Von den Thesen D.s seien nur die zwei wichtigsten, auch für Nichtfachleute interessanten, referiert: D. rechnet mit der Existenz eines Erzählwerkes, das von der frühen Königszeit handelt. Es umfaßt 1 Sam 9-1 Kön 2 (eventuell 1 Sam 1-1 Kön 12); es stammt aus dem 8. oder 7. Jh. In 1 Kön 17-2 Kön 10 ist nach D. ein »vor-dtr prophetisches Erzählwerk >Über den Kampf Jhwh's gegen Baal« (249) aufgenommen worden, das im wesentlichen um die »Auseinandersetzung zwischen Propheten und Königen«, näherhin »die Alleinverehrung Jhwh's« (ebd.) kreist. Es gehört ins 7. Jh. und dürfte unter Manasse verfaßt worden sein. Seine Einarbeitung in DtrH erfolgte durch DtrP, der als »ehrlicher Makler« (250, eine Formulierung M. Noths), als Vermittler zwischen historiographischer und prophetischer Tradition wirkte. »DtrP wollte (deuteronomistisch) die Alleinverehrung Jhwh's und (prophetisch) die Distanz zu staatlicher Macht und die unbedingte Verlässlichkeit des Gotteswortes betonen.« (ebd.) DtrP, der, wie D. selber feststellt, unter die Räder des Kompromisses zwischen Göttinger und CrossSchule zu geraten droht (237), ist also auch ein idealer Theologe.

D. schreibt ausgezeichnetes, leicht lesbares Deutsch - mit einem leichten Hang zum Journalistischen. An einigen Stellen versucht er, durch gewagte Formulierungen die Leser auf seine Seite zu ziehen. Gelegentlich weiß D. wie Stefan Heym, dem er diesen Vorwurf macht - etwas zu viel. Auf S. 49 etwa redet er von der »ausdifferenziert[en] « Sprache der Thronfolgeerzählung; den Nachweis dafür und ein Beispiel für nicht ausdifferenziertes Hebräisch bleibt er jedoch schuldig. Das Urteil über den »genialen JehuNovellisten« (169), den Verfasser der Grundschicht von 2 Kön 9 f., mag 
berechtigt sein; da es so steil ist, bedürfte es einer ausführlichen Begründung. Oder 167: Unter den Omriden »standen Thron und Altar (wie später noch so oft) nah beieinander«. Wäre ihre Trennung das (Normale oder) Ideale gewesen? Und: Was soll die Klammerbemerkung? 177: Wenn Manasse »bedingungslos treuer assyrischer Vasall« war, so doch auch einer der politisch bedeutendsten Könige Judas. 191: „Gerade an dieser Stelle rückt das klassisch orientalische do-ut-des-Verhältnis zwischen Gott und König ... bedenklich [!] nahe.« Bildet dieses nicht auch Grundlage des Alten Testaments?

Wenn D. - gelegentlich hinreißend - nacherzählt, kann man (wie bei G. von Rad) den Eindruck gewinnen, dabei zu sein; der garstige historische Graben zwischen Vergangenheit und Gegenwart schließt sich. Seine Aufsätze können deshalb fundamentalistisch mißverstanden werden. Rez. las D.s Aufsätze auch mit den Augen eines milden Pietisten und eines sozial und politisch engagierten Christen / Theologen - und kam jedesmal in je unterschiedlicher Weise auf seine Rechnung. Eine faszinierende und herausfordernde Theologie, bei der all dies möglich ist.

3. James Loader, der lange Jahre an südafrikanischen Universitäten wirkte und seit 1997 Professor für Altes Testament an der Evangelischtheologischen Fakultät der Universität Wien ist, legt den Sammelband mit den meisten und thematisch am breitesten gestreuten Aufsätzen vor. L. will diese in der Mehrzahl auf Englisch verfaßten und jetzt ins Deutsche übersetzten, teilweise abgelegen publizierten Arbeiten deutschsprachigen Lesern zugänglich machen. Für die Neupublikation vereinheitlichte er sie formal und erstellte ein einziges Literaturverzeichnis.

$\mathrm{Da}$ L. nicht in einer der großen traditionellen Schulen Europas (oder Amerikas) groß wurde, obwohl er sich auch in diesem Milieu bewegt hat, merkt man seinen Aufsätzen gut an. Seine Gesprächspartner sind neben Fachexegeten auch recht stark Systematiker; interessiert ist er auch an Literatur und - besonders stark - an Musik. L.s Studium der Semitistik und Altphilologie schlägt sich in seinen Arbeiten kaum nieder. Der Titel für den Sammelband (»Begegnung mit Gott«) wird diesem gerecht. Die Thematik, die L. stärker als jede andere beschäftigt, lautet: Wie wirken Gott und Mensch zusammen?

Wegen der Themenvielfalt des Aufsatzbandes sollen die (meisten) Beiträge, häufig ohne Nennung des Titels, einzeln vorgestellt werden - durch Hinweis auf eine in ihnen entfaltete These, durch die Inhaltsangabe oder eine Einzelheit, die dem Rez. ins Auge sprang. Koh 3,2-8 ist ein Sonett. Die korrekte Übersetzung des schwerverständlichen Verses Ruth 2,17 lautet: »Dies ist ihre Bleibe, in einem gewissen Sinn ihr Haus.« (17) - Der Pen- 
tateuch kann verschieden gelesen werden: als »Biographie des Mose«, als »Wanderbericht Israels zum verheißenen Land« oder als »Erzählung über Israels Tora«, »in der die Geschichte vom ethno-biographischen Epos Israels gerahmt wird « (25). In seiner Mitte steht Leviticus, dessen Zentrum seinerseits Lev $16 \mathrm{f}$. bildet; interessanter- oder typischerweise geht L. nicht auf diese Kapitel ein. Schlägt hier protestantisches Erbe durch? - Ausgehend von einer »Falschübersetzung« G. von Rads in seinem Kommentar zu Gen 45,5 zeigt L. auf, daß göttliche Führung und menschliche Verantwortung einander nicht ausschließen. - Das Büchlein Ruth ist, wie motivgeschichtliche Beobachtungen zeigen, eine Patriarchen-, respektive Matriarchengeschichte. - Was im Hause Davids passiert, ist in dem Haus Elis präfiguriert. An beider Beispiel wird gezeigt: "Was er [Gott] gesagt hat, muß nicht passieren. Was er nicht gesagt hat, kann passieren. Denn er ist Gott.« (66) In »Jedidja oder Amadeus« verhandelt L. wieder das Thema, wie göttliches und menschliches Wirken sich zueinander verhalten. Der Vergleich mit Prov und Est zeigt, daß die Thronfolgegeschichte, deren Abfassung in die Zeit nach dem Fall Samarias fällt, weisheitlich geprägt ist. - In seinem zweiten Hiobaufsatz bemüht sich L. um den Nachweis, daß Gottes Gerechtigkeit von allen moralischen Widersprüchen des menschlichen Lebens unberührt bleibt. »Die Antwort [auf das Hiobproblem] enthält daher ein Rätsel: Es schließt die Erkenntnis eines irrationalen Elements ein - der Transzendenz Gottes wie der Gemeinschaft mit Gott.« (132) - Aus L.s Ausführungen zu Jona 4,2-3 sei ein schöner Satz zitiert, den Rez. noch immer nicht richtig einzuordnen versteht: »Jona beklagt sich bei Gott, daß es Gott gibt.« (147) Johannes Pedersens Machtbegriff wird nach L. den alttestamentlichen Tatbeständen nicht gerecht. - »Gerste, Bullen, Land und Levirat«: Unter diesem Titel geht L. dem Verhältnis zwischen Erzählung und Gesetzen anhand der Ruthnovelle nach (die jenen zeitlich vorangeht). - Der Aufsatz »Weise rufen zur Ordnung« enthält eine Abrechnung (ein fast zu milder Ausdruck) mit Westermanns Weisheitsbuch, der Aufsatz »Lernen im Indikativ« mit dessen These, wonach Weisheits- und Mahnspruch scharf voneinander zu trennen seien. - In »Weisheit aus dem Volk für das Volk« heißt L.s Opponent Golka; er setzt sich mit dessen Interpretation afrikanischer Weisheitssprüche auseinander. L. warnt vor einer vorschnellen Verortung von Weisheitssprüchen am Hof oder im Volk. - Der Aufsatz »Das Vorbild des priesterlichen Segens in IQS« [II,2-4] enthält eine formale Analyse dieses Textes, der auf Num 6,24-26 aufbaut, und bietet einige wenige inhaltliche Ausführungen. Antiochenische und alexandrinische Auslegung der Heiligen Schrift stehen einander nicht so diametral gegenüber, wie oft vertreten. Diese Behauptung stellt L. im Zusammenhang mit der Analyse von sechs Chrysostomospredig- 
ten über Jes 6 auf. - Am Beispiel von Johannes Brahms' Opus 121 (Vier ernste Gesänge) zeigt L. auf, wie Künstler mit biblischen Traditionen umgehen: eklektisch und phantasievoll. - Es ist von der »Kuenen-These«, nicht von der »Graf-These « zu sprechen; denn Kuenen und nicht Graf, wie in der Forschungsgeschichte gern behauptet, verdanken wir die »Entdeckung einer exilischen Priesterschrift, in welche die Gesetze in den Zusammenhang eingeführt wurden« (283). Diese temperamentvoll vorgetragene These verbindet L. mit der Klage, dieser holländische Alttestamentler werde stark unterschätzt. Nicht überall, darf man hinzufügen, ohne für diese Behauptung einen Beleg liefern zu müssen. - Von den systematischen Beiträgen sei nur der gewichtigste und anregendste referiert, »Logos spermatikos«. L. plädiert in ihm für theologische Beschränkung und Bescheidenheit. Neben großen Religionsgeschichten und Theologien des Alten Testaments - letztere notgedrungen wie zu Recht einseitig - muß es auch Platz für »theologische Miniaturkunst« (339) geben, d.h. für thematisch eingeschränkte Untersuchungen. In ihnen kommt von »Mitte-Theologien« Übersehenes und Vernachlässigtes zu seinem Recht. Das Überschaubare, so L., ergreift den Menschen womöglich gleich stark wie »massive Eindrücke« (337). Jeder Topos kommt so zu seinem Recht und wird nicht von anderen zur Seite geschoben. Theologische Miniaturkunst nimmt weiter das Mißtrauen ernst, das die Postmoderne "großen Schemata und (offenbarungs-)positivistischen Sicherheiten« (338) entgegenbringt. Ein Aufsatz, bei dessen Diskussion im Seminar mit Sicherheit heftige Kontroversen entbrennen werden! - Im letzten Beitrag des Sammelbandes, »Narren erklären, Weise nicht«, stolperte ich unter anderem über folgenden Satz: »Die Geschichte der Alttestamentlichen Wissenschaft ist übersät mit Trümmern aus Glauben und Frieden, entstanden aus den Hypothesen über den Ursprung des Pentateuch.« (345) Das Buch (und insbesondere die Übersetzungen aus dem Englischen) sind zum Glück wesentlich besser als dieser Ausrutscher, der hier stellvertretend für vergleichbare zitiert wurde.

4. Rudolf Mosis’ „Gesammelte Aufsätze zum Alten Testament« enthalten ausschließlich klassische Exegesen. Nur mit dem Aufsatz »Gen 15,6 in Qumran und in der Septuaginta«, der als einziger der zwölf Beiträge erstmals in diesem Bande publiziert ist, überschreitet M. diese Grenze geringfügig. M., dessen Untersuchungen noch nicht die ihnen gebührende Aufmerksamkeit gefunden haben, arbeitet sehr klassisch und sehr solide. Gerne geht er von einer offenen Frage, einem ungelösten Problem aus, das er beharrlich einer Lösung zuführt. M. argumentiert so, daß man ihm beim ersten Lesen seiner Arbeiten immer zustimmt - ich wenigstens -, mehr als bei anderen Exegeten. Dem Charakter seiner Arbeiten entsprechend werden 
im folgenden - weitgehend unkommentiert - deren Thesen referiert, ausführlicher die besonders profilierten. Statt der Aufsatztitel nennen wir meist nur die behandelte Bibelstelle.

Gen 9,1-7: V. 1-4, die sich in der Abfolge der Elemente mit Gen 1,28-30 berühren (Mehrungssegen für den Menschen, Bestimmung seines Verhältnisses zu den Tieren, Regelung der Nahrung) gehören zur Grundschrift des priesterschriftlichen Werkes $\left(\mathrm{P}^{\mathrm{G}}\right)$. Der Abschnitt steht an der Stelle, die in anderen Sintflutgeschichten das Opfer einnimmt. V. 2 berichtet von einem Heiligen Krieg der Menschen gegen die Tiere, welche die in der Nachsintflutzeit aggressiv tätigen Mächte des Chaotischen und Bösen repräsentieren. V. 4 enthält ein Speisegebot. Gen 9,1-4 zeichnet die Realität, Gen 1,28-30 im Unterschied dazu die "Erinnerung an das noch ausstehende [Glück]« (54). - Gen 15,6 muß wie folgt übersetzt und interpretiert werden: \Und da er nun schon immer im gläubigen Vertrauen auf Jahwe lebte, achtete er es [sc. die Verheißung Jahwes] für sich als eine heilswirkende, rechte Tat.« (89) - »Gen 15,6 in Qumran und in der LXX«: Die Übersetzung von Gen 15,6 durch die LXX (Und er [Abraham] glaubte, und es wurde ihm zur Gerechtigkeit angerechnet) bildet nicht Interpretation des Übersetzers, sondern geht auf eine hebräische Vorlage zurück, die, allerdings bruchstückhaft,

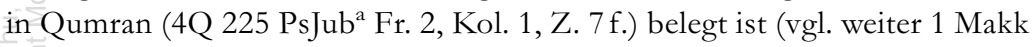
2,52; Ps 106,31; Jub 14,6; 31,23; Targum Neofiti). Es handelt sich um ein klassisches Tiqqun sopherim (Korrektur eines Textes aus theologischen Bedenken): Abraham kann Gott dessen Tun nicht in irgendeiner Weise anrechnen. So könnte MT, der die ältere Textform bietet, in seinem Zusammenhang gelesen werden. - Ex 19,5b.6a: Jahwe sieht Israel als Krongut (סגלה), als priesterliches Königreich (ממלכת כהנים) und heiliges Volk (גוים) an. Diese Ausdrücke bezeichnen, was Israel von Jahwe erhalten hat, nicht Funktionen, die es den fremdenVölkern oder Jahwe gegenüber wahrnehmen müßte. - Jes 40,6-8: bildet entgegen einer beliebten These keine Zusammenfassung von Jes 40-55. M. schlägt folgende Neuinterpretion vor: Auf die Aufforderung einer Stimme hin, als Rufer aufzutreten, antwortet ein Ich (der Prophet) mit dem Hinweis auf die Vergänglichkeit allen Fleisches. Darauf repliziert die Stimme, der דבר יהוה komme zustande für immer, und darin werde dem Volke Bestand geschenkt für immer. Der Prophet lebe, so M., in verzweifelter Zeit (?, Rez.), in ihr würden Hinfälligkeit und Aussichtslosigkeit überwunden durch das Heilswort Gottes, »das zustande kommt für immer« (171). - Jer 3,21-4,2: Exegese. - Ez 14,1-11: Dieser Ruf zur Umkehr ist nicht unmittelbarer Niederschlag mündlicher Verkündigung, sondern von Anfang an als literarische Komposition konzipiert. V. 1 bildet nicht eine historische, sondern typische (paradigmatische) Situation ab. Ein wichtiger 
Übersetzungsvorschlag zu Jer 20,7 (210): »Du hast mich zu einem Narren gemacht« (nicht: »Du hast mich betört«). - Hos 2,16f.: Hos 2,10-15, ursprünglich ein Gerichtswort, in der »jetzigen Gestalt jedoch ... klagende Rede Jahwes ..., in der ... die Verlorenheit Israels mit einer gewissen Endgültigkeit fest[ge]stellt und beklagt« (270) wird, enthält durch V. 16 f. eine Antwort: Jahwe wird durch sein heilvolles Handeln an Israel dieses befähigen, ihm »hör- und lernwillige[r] Partner« (271) zu werden, so daß die neu einsetzende Geschichte nicht zum Scheitern verurteilt ist. Ein Eintrag fürs Wörterbuch: פתה Pi. bedeutet in Hos 2,16: »jemanden in den Zustand bringen, in dem er helfende Unterweisung und erziehende Führung annehmen kann und will« (263). - Psalm 23: Gutes Referat des neueren Verständnisses des Psalms mit wenig Verweisen auf Sekundärliteratur und spärlichen Fußnoten. M. zeichnet drei wichtige Interpretationslinien nach: Das Bild vom (guten) Hirten, lange Zeit von der Lebenswelt des Beters her gedeutet, spielt erst vom babylonischen Exil an eine wichtige Rolle. Es dient dazu, den »Weg aus der Verlorenheit unter die Völker ... zurück zum Ort seiner Gegenwart, zum Haus Jahwes, zum Zion« (293) abzubilden. »In diesen exilischen Heilsweg ist aufgenommen der alte Heilsweg aus dem Sklavenhaus Ägypten ... zum gelobten, verheißenen Land, zu seiner Stadt und zu seinem Tempel.« (ebd.) Von einem Fest- und nicht einem Kultmahl handelt der zweite Teil des Psalms. Hirten- wie Gastgebermetapher sind Ausprägungen der Königsmetapher. Bei der Lektüre der Heiligen Schrift gewinnt der Beter Mut dazu, die Identität seines Heilsweges mit dem des Volkes zu bekennen. - Ps 51,20f. (Die Mauern Jerusalems). Aus diesem thesenreichen Beitrag sei nur das Allerwichtigste referiert: Die beiden Verse dieses Psalms, der von Ez 36 abhängen dürfte, sind mit Sicherheit nach dem Mauerbau unter Nehemia verfaßt worden. Es geht in ihnen um die Erwartung »eines schlechterdings neuen, irdisch-himmlischen Jerusalem ..., und dieses neue Jerusalem signalisiert hier die schlechterdings nicht mehr überbietbare und die nicht mehr verlierbare Vollendung Israels und der Welt« (310). Als »Ikone« »für das ungestörte und nicht mehr zerstörbare [corr.] Verhältnis zwischen denen, die Opfer darbringen, und dem, dem sie dargebracht werden, zwischen Israel und seinem Gott« werden die »Opfer der Gerechtigkeit« dienen (315f.). - Ps 93: Dieses Bekenntnislied (kein Hymnus!) zerfällt in zwei Teile, V. 1 und V. 2-5. Der erste Teil gibt das Doppelthema des Psalms an (Festigkeit der Welt aufgrund der königlichen Herrschaft Jahwes). Gerahmt von V. 2 und 5 führt der Hauptteil, V. 3 f., das Doppelthema von V. 1 in seiner Dramatik für den Beter aus. Die Frage nach der Gliederung, und, damit verbunden, seiner inhaltlichen Akzentuierung ist stark umstritten. M. macht folgenden Vorschlag: יהוה מדך ist Mottosatz, auf 
den ein Trikolon folgt, dessen letztes Wort mit Qumran als והתאזר zu lesen ist:

\author{
In Hoheit ist gekleidet \\ gekleidet ist Jahwe in Macht \\ und hat sich umgürtet. (334)
}

Auf dem dritten Stichos - Jahwe gürtet sich zu Krieg und Kampf (er zieht nicht nur einen feierlichen Ornat an) - ruht der Nachdruck. - Ps 126: Dieser Psalm hängt vom Joelbüchlein $a b$, das M. ins 5./4. Jh. datiert. V. $5 \mathrm{f}$. beschreiben die gegenwärtige Situation, V. 1-3, die nicht mit einem bestimmten Ereignis in Zusammenhang stehen, die zukünftige. V. 1b-3 bilden Apodosis zuV. 1a. »Sein wie Träumende« charakterisiert wie »voll Freude« die Zukunft. »Wie Träumende« statt »Träumende« wie in der Vorlage bei Joel schreibt der Verfasser von Ps 126, um dem Mißverständnis vorzubeugen, es ginge um »eine[.] prophetische[.] Offenbarungsvermittlung an andere« (380).

5. Ludwig Schmidt gehört zusammen mit A. Graupner zu den wenigen Aufrechten, die weiterhin an der Existenz einer elohistischen Quellenschrift festhalten. Das sollte allerdings kein Grund sein, die von Otto Kaiser angeregte Sammlung von elf Beiträgen (drei davon erstmals publiziert) zum Pentateuch ungelesen zur Seite zu legen oder den nun folgenden Abschnitt zu überspringen. Sch.s literarkritische und redaktionsgeschichtliche Analysen liest mit Gewinn auch, wer seine Voraussetzungen nicht teilt.

Sch. sah sich, wie er im Vorwort seiner Aufsatzsammlung festhält, zu keiner grundlegenden Revision seiner Ansichten gezwungen, höchstens zu Retuschen in Einzelpunkten. Dementsprechend setzt er sich mit neueren Ansätzen nur marginal auseinander.

Ein biblischer Text beschäftigt Sch. immer wieder (am ausführlichsten in seinem ersten Beitrag): Gen 12,1-3. Umso mehr befremdet, daß er Crüsemanns wichtige These zu dieser Stelle ${ }^{1}$ - Gen 12,1-3 bildet nicht Bestandteil von J, sondern ist Brücke zwischen Ur- und Vätergeschichte - nach 1981 nicht zur Kenntnis genommen hat - oder der Kenntnisnahme für nicht wert hält.

Im Aufsatz »Israel ein Segen für die Völker?« setzt Sch. dieser These H.W. Wolffs ein dezidiertes Nein entgegen. J ist auch sonst jedes Sich-selbstRühmen des Menschen fremd. In diesem wie im folgenden Aufsatz (»Überlegungen zum Jahwisten«) vertritt Sch. die Meinung, J habe sein planvoll angelegtes Werk unter Salomo verfaßt; J betrachtet dessen Reich, das seine

${ }^{1}$ F. Crüsemann, Die Eigenständigkeit der Urgeschichte. Ein Beitrag zur Diskussion um den »Jahwisten«, in: Die Botschaft und die Boten. FS für H. W. Wolff zum 70. Geburtstag, hg.v. J. Jeremias u. L. Perlitt, Neukirchen 1981, 11-29. 
Existenz Jahwe allein verdankt, als Erfüllung der an Abraham ergangenen Verheißung. - Viel Literarkritik und Redaktionsgeschichte enthalten die nächsten drei Beiträge, der erste auch überlieferungsgeschichtliche Überlegungen: »Der Kampf Jakobs am Jabbok (Gen. 32,23-33)«, »Die alttestamentliche Bileamüberlieferung« und »Jakob erschleicht sich den väterlichen Segen. Literarkritik und Redaktion von Genesis 27,1-45«. In den Beiträgen zu Bileam und Gen 27 spielt der Elohist eine prominente Rolle. - Zu »Väterverheißungen und Pentateuchfrage«: Das um 550 v.Chr. verfaßte Werk DtrH kennt die Landverheißung an die Väter, wie sie in Gen 12,7 und 28,13 belegt ist. Nach Sch. lag also spätestens 587 v.Chr. ein Werk vor, das von Abraham und Jakob handelte. Er betont in seinem 1992 (!) publizierten Aufsatz mit Nachdruck, dieses Resultat hänge nicht von einem bestimmten Modell der Pentateuchentstehung ab. In »El und die Landverheißung in BetEl« schlägt Sch. eine gegenüber bisherigen Vorschlägen leicht modifizierte Quellenscheidung vor; die beiden Versionen des Textes, J und E, enthalten eine je eigene Gestalt einer Ätiologie des Heiligtums Beth-el. - Zu »Weisheit und Geschichte beim Elohisten«: Der Heilsgeschichte »ist das Thema Gottesfurcht so zugeordnet, daß jene Menschen, die die besondere Zuwendung Gottes erfahren haben, ihrerseits von jener Gottesfurcht bestimmt sein müssen, die nicht von einer besonderen Gottesbeziehung abhängig ist« (166). Zwei für Sch.s Position und Argumentationsweise bezeichnende Sätze seien hier kommentarlos wiedergegeben: »Damit hat die Heilsgeschichte, die der Elohist in eigener Weise entwickelt ${ }^{73}$, ein selbständiges Gewicht.«Anm. 73: »Das bestätigt, daß E eine eigene Quellenschrift ist.« (166) - Nur durch ein Zitat sei der Aufsatz »Die Darstellung Isaaks in Genesis 26,1-33 und ihr Verhältnis zu den Parallelen in den Abrahamerzählungen« vorgestellt: »Für die Frage der Entstehung des Pentateuch ist aber wichtig, daß Gen 26* und die parallelen Abrahamerzählungen in Gen 20 und 21,22ff zeigen, daß es die Quellenschriften des Jahwisten und des Elohisten gegeben hat und, daß E von J literarisch abhängig ist.« (223) - Nicht erstaunt der leicht apologetische, selbstverständliche und deshalb fast überflüssige Satz am Schluß von »Diachrone und synchrone Exegese am Beispiel von Exodus 3-4«: »Wie schon an der jehowistischen Redaktion von Ex 3,1-4,17 wird somit auch an dem späten Abschnitt Ex 4,20b-23 deutlich, daß die diachrone Analyse der Texte für die Interpretation der Redaktionen und der Endgestalt des Pentateuch von erheblicher Bedeutung ist.« (250) - Zum letzten Aufsatz, »Mose, die 70 Ältesten und die Propheten in Numeri 11 und 12«: Die Ältestenschicht von Num 11, die in der Exilszeit, vielleicht vom Jehowisten, in die Wachtelerzählung eingefügt wurde, dient dem Zweck, nach dem Ende des Staates Juda die Ältesten als Führer des Volkes zu legitimieren. Erst die 
Schlußredaktion des Kapitels setzt diese Ältesten auch zur Prophetie in Beziehung. Die Endredaktion von Num 12 hebt Mose von Propheten und Priestern ab und streicht seine Einzigkeit heraus: Jahwe redet mit ihm von Mund zu Mund und in klaren Worten, und deshalb ist seine Botschaft der der Propheten überlegen. 